OPEN ACCESS

Edited by:

Lino Nobili,

University of Genoa, Italy

Reviewed by:

Lyudmila S. Korostovtseva, Almazov National Medical Research

Centre, Russia

Marina Sorrentino Hernandes,

Emory University, United States

Julian Nicolas Acosta,

Yale University, United States

*Correspondence:

Ekaterina Spektor

ekaterina.d.spektor@gmail.com

Specialty section:

This article was submitted to

Sleep Disorders,

a section of the journal

Frontiers in Neurology

Received: 25 April 2021

Accepted: 17 August 2021

Published: 27 September 2021

Citation:

Spektor E, Fietze I and Poluektov MG (2021) Periodic Limb Movements Syndrome in Patients With Cerebral Small Vessel Disease: Protocol for a Prospective Observational Study.

Front. Neurol. 12:700151. doi: 10.3389/fneur.2021.700151

\section{Periodic Limb Movements Syndrome in Patients With Cerebral Small Vessel Disease: Protocol for a Prospective Observational Study}

\author{
Ekaterina Spektor $^{1 *}$, Ingo Fietze ${ }^{2,3,4}$ and Mikhail G. Poluektov ${ }^{1}$ \\ ${ }^{1}$ Department of Sleep Medicine, Chair of Neurology and Neurosurgery, University Clinical Hospital No. 3, I.M. Sechenov First \\ Moscow State Medical University, Moscow, Russia, ${ }^{2}$ Center of Sleep Medicine, Charité - Universitätsmedizin Berlin, Berlin, \\ Germany, ${ }^{3}$ The Fourth People's hospital of Guangyuan, Guangyuan City, China, ${ }^{4}$ The Federal State Autonomous \\ Educational Institution of Higher Education, I.M. Sechenov First Moscow State Medical University of the Ministry of Health of \\ the Russian Federation, Moscow, Russia
}

Background: Cerebrovascular diseases are the leading cause of cognitive decline and dementia. Therefore, the investigation of the potential ways to slow down the disease progression is an important research field. Periodic limb movements in sleep (PLMS) are known to be associated with transient changes in heart rate and blood pressure. These changes might influence the course of cerebral small vessel disease (cSVD). Nevertheless, the clinical significance of PLMS, particularly its influence on cardiovascular diseases course, is still controversial and underinvestigated.

Methods/design: Patients from 60 to 75 years old diagnosed with cSVD will undergo nocturnal polysomnography. Subjects with apnea/hypopnea index under 5 will be enrolled. Sleep quality and daytime functioning will be assessed at baseline with self-reported questionnaires. Brain MRI and cognitive assessment will be performed at baseline and in the 2-year follow-up. Progression of cSVD markers and cognitive dysfunction will be compared between patients with PLMS index (PLMI) equal to or more than 15 movements per hour of sleep and controls (PLMI < 15/h).

Discussion: The negative role of PLMS in CSVD progression and related cognitive decline is expected. We suppose that patients with PLMS tend to worsen in cognitive performance more rapidly than age-, gender-, and comorbidity-matched controls. We also expect them to have more rapid white matter hyperintensities and other cSVD marker progression. The limitations of the study protocol are the short follow-up period, the absence of a treatment group, and inability to make a conclusion about causality.

Keywords: aged, cerebral small vascular diseases, cognitive dyfunctions, magnetic resonace imaging (MRI), polysomnogaphy, periodic limb movement, sleep

\section{INTRODUCTION}

\section{Background}

Cerebrovascular diseases (CVD) remain a global problem today because of high prevalence and related cognitive impairment. Increase in vascular dementia rate is the current trend worldwide. Thus, the identification of new predictors for CVD seems to be an important medical and social problem. Cerebral small vessel disease (cSVD) is one of the most prevalent forms of CVD. This term is used to describe clinical features and structural changes in brain tissue occurring due to damage 
of small perforating arterioles as well as capillaries and venules. This vascular pathology results in white tissue and basal ganglia lesion that leads to neurological deficit and cognitive decline (1). cSVD is considered to be the leading cause of cognitive dysfunction and dementia (2-4). Moreover, cSVD is associated with an increasing risk of cerebral infarct and intracranial hemorrhage $(5,6)$. However, cSVD is a broad term, and it encompasses "pure cSVD," Binswanger's disease, and mixed dementia (when Alzheimer's disease overlaps with cSVD), which are inherited forms of cSVD (7).

Although there are generally accepted risk factors for cSVD including hypertension, diabetes, and aging (1), recent researches have demonstrated a significant role of sleep disturbances in cSVD progression. The significance of obstructive sleep apnea is now well-established and extensively reported (8, 9). Nevertheless, there is a growing body of literature that recognizes the negative influence of other sleep disorders, including periodic limb movements (LMs) in sleep (PLMS), on the course of cardiovascular diseases and CSVD in particular. A probable explanation of this impact is a sleep fragmentation that results in episodes of heart rate increase and blood pressure elevation (8-12).

PLMS is a condition characterized by periodic episodes of repetitive and stereotypic flexions of the hips, knees, and ankles (less common-arms movements) that manifests during the sleep. Diagnosis of periodic limb movements disorder (PLMD) requires conformity with two criteria. Firstly, periodic limb movements index (PLMI) $>5 / \mathrm{h}$ in children and $>15 / \mathrm{h}$ in adults must be detected by polysomnography (PSG). Second, PLMS in sleep must lead to clinically significant sleep disturbance or impairment in different relevant areas of functioning and cannot be better explained by another current sleep, medical, or neurological disorder such as narcolepsy, restless leg syndrome (RLS), or REM sleep behavior disorder $(13,14)$. The prevalence of PLMS worldwide, including the Russian population, is estimated to be $3.9-6 \%$ in the general population and around $34 \%$ in aged patients (above 60 years) $(15,16)$.

PLMS are described to be associated with various conditions, such as congestive heart failure (17), alcohol abuse (18), Tourette syndrome (19), syringomyelia (20), multisystem atrophy, and Parkinson's disease (21). Increased PLMS are also observed in a significant proportion of patients with various sleep disturbances (narcolepsy and obstructive sleep apnea). Moreover, this phenomenon is also detected in healthy subjects, and the prevalence increases with age $(13,16,22)$. The closest association of PLMS is found with RLS. Approximately $85 \%$ of patients with RLS show high PLMI during nocturnal sleep $(13,22)$.

\section{Pathophysiology and Cardiovascular Effects of PLMS}

Initially, PLMS were studied in close connection with RLS. These conditions are supposed to share common pathophysiological mechanisms since E. Lugaresi et al. showed that most RLS patients had periodic leg movements during sleep $(23,24)$. RLS is defined by the International Classification of Sleep Disorders (III Edition) as the syndrome characterized by an urge to move the legs, sometimes accompanied by an uncomfortable sensation that occurs primarily with rest/inactivity; it is partially or totally relieved by movement, for as long as the movement occurs, and occurs primarily in the evening or at night (14). Despite close relations between PLMS and RLS, they are considered to be different neurological processes since one of them can occur in the absence of the other (23), although the precise origin of PLMD and RLS is not established. Probably, dopamine or its derivatives play the primary role in these states because a high prevalence of both RLS and PLMS is observed in Parkinson's disease patients (25). Moreover, worsening of symptoms is observed after dopamine antagonist intake and vice versa; remarkable improvement can be achieved by dopaminergic agent intake (26). On the other hand, Manconi et al. investigated the role of dopamine using pharmacological agents and revealed that RLS can be reduced using both dopaminergic and nondopaminergic agents, whereas PLMS respond to dopaminergic agents only (27). Thereby it is likely, that PLMS is under stronger dopaminergic control in comparison with RLS.

The dysfunction of descending inhibitory pathways with subsequent disinhibition of the segmental apparatus of the spinal cord is believed to be the reason for PLMS (28). The D2 and D3 receptors on the preganglionic neurons of the lateral horns are considered to be involved (29). Probably, the diencephalic dopaminergic structures (A11 neuronal group) take part in the genesis of PLMS and RLS because the A11 diencephalospinal pathway is crucial for pain control and sensorimotor integration at the spinal level (30). For example, the study on rats showed the presence of A11 dopaminergic terminals in posterior spinal horns and their vicinity to the motor neurons of anterior horns (31). The model involving the crucial role of dopamine could explain the positive effect of intake of dopaminergic agents in patients with both PLMS and RLS.

The subcortical origin of PLMS is confirmed by the experiment with paired-pulse transcranial magnetic stimulation (ppTMS). Rijsman et al. revealed decreased intracortical inhibition and increased intracortical facilitation while the cortical excitability was normal in subjects with RLS and PLMS. These aberrations appeared more significant in the leg that was more disturbed with sensorimotor symptoms and had higher PLMI than the other leg (28). The presumption about the supraspinal subcortical origin of PLMS is confirmed by studies in patients with cervical or thoracic traumatic complete spinal cord injury. They discovered higher PLMI in comparison with healthy controls. The probable explanation is the damage of descending pathways and loss of the supraspinal inhibitory influence (32, 33). It is also confirmed indirectly by the investigation of $\mathrm{H}$ reflex recovery curve in PLMD patients. The results of this study demonstrated increasing late facilitation and decreasing late inhibition in comparison with controls (28). These findings might reflect decreased postsynaptic inhibition.

There is evidence that PLMS provokes a considerable increase in sympathetic activity throughout sleep $(34,35)$, which results in heart rate and blood pressure increase $(9,36)$. The repeated increase in nocturnal heart rate and blood pressure are considered to be the reason for the negative influence of PLMS on the cardiovascular system. Nevertheless, some researchers 
deny the existence of persistent sympathetic hyperactivity in the absence of movement (in other words, sympathetic hyperactivity exists only when PLMS occurs) $(37,38)$. On the other hand, other authors highlighted the primary role of the sympathetic nervous system in the generation of PLMS (39). Thus, there is no consensus on whether the PLMS itself can change the autonomic tone.

PLMS is often associated with EEG arousals. It is not yet established whether one phenomenon is the reason for the other, or both phenomena have the same origin. However, the latter seemed more likely $(22,40)$. The effect of arousals on daytime consequences is controversial. The high frequency of PLMS with arousals during sleep could be a hypothetical trigger for nocturnal arrhythmias and hypertension (41), and PLMSrelated arousals may be a risk factor of ventricular arrhythmia (42). However, the majority of the researchers do not emphasize the difference between the PLMS with arousals and the PLMS without arousals. There are data showing that PLMS frequency (with and without arousals) is associated with a significant increase in systolic blood pressure $(10,43)$.

However, evidence of the relationship between PLMS and cSVD is scarce. The prevalence of PLMS in the cSVD subpopulation was not evaluated. Coelho et al. showed that patients with a history of stroke had a greater prevalence of PLMS and significantly higher mean PLMI than controls (44), but according to the research made by Manconi et al., PLMS are equally frequent in patients with a history of stroke or TIA and the general population (45). There are data about a higher prevalence of RLS in patients with cardiovascular diseases (7.7$36 \%$ ), which is three times higher than the prevalence of RLS in the general population (46). The cardiovascular effects of RLS are suggested by some researchers to be mediated by increased PLMI (47).

Current evidence suggests that there is repetitive sympathetic activation accompanying PLMS during night sleep. This phenomenon could be a potential link between PLMS and negative impact on the cardiovascular system (36). The presumed cardiovascular effects of PLMS could underlie reported associations of higher PLMI with arterial hypertension severity (48), the incidence of cardiovascular events (49), increased risk of death in patients with systolic dysfunction following acute decompensated heart failure (50), and mortality risk in patients with systolic heart failure (12). It is reasonable to assume that PLMS-related activation of the sympathetic nervous system could be an explanation of the negative role of PLMS in CVD course, although it remains an underinvestigated issue. It is shown in cross-sectional studies that the severity of PLMS is associated with the extent of white matter hyperintensities (WMH) in patients with cSVD $(51,52)$ and in patients with first-ever minor stroke or transient ischemic attack (53). On the other hand, it is known that any pathological state accompanied by sleep fragmentation (sleep apnea, PLMS, and insomnia) may increase the risk of vascular diseases (9). Therefore, a more general process could be responsible for increased cardiovascular risk in PLMS patients.

Another unresolved question is the relevance of PLMS to cognitive dysfunction. Whereas, PLMS could worsen the course of cSVD, the greater cognitive decline of PLMS patients is expected. However, only a few studies focused on this issue, and their results suggest a positive correlation between the PLMI and cognitive decline. Significant association of higher PLMS frequency with impaired executive function was demonstrated among older men without dementia (54), patients with Parkinson's disease independent from psychomotor speed (55), and patients with cSVD (52). Besides, worse sleep efficiency has been also shown to be associated with poorer performance in tasks evaluating the executive domain (56), but the influence of PLMS on sleep quality and its ability to lead to sleep fragmentation is disputable. Moreover, some authors report that age-related changes in sleep architecture with a decline in slow-wave sleep might mediate age-related decline in executive control (57). It appeared confusing since the prevalence of PLMS increases with age too.

On the contrary, the cognitive impairment in CSVD is fully investigated, and it involves similar changes in executive functions, attention, language fluency, and set shifting $(58,59)$. MRI features of cSVD are summarized in STRIVE criteria (STandards for ReportIng Vascular changes on nEuroimaging). They consist of WMH, recent small subcortical infarcts, lacunes, cerebral microbleeds, and perivascular spaces (4). Substantial contribution to cognitive decline has been shown for frontaland temporal-located cerebral microbleeds (60), progression of periventricular WMH (61), accumulation of lacunar infarctions (58), and perivascular spaces in combination with other markers (62).

\section{Rationale}

The determination of the role of PLMS in the progression of cSVD MRI features and increase of cognitive dysfunction would create a new approach to patient management. Confirming of the negative impact of PLMS on the course of CSVD makes considerable further investigation the utility of the pharmacologic correction of PLMS in future studies. Currently, there is no generally accepted point of view on clinical significance of PLMS and its exact connection with both cSVD course and cognitive decline.

\section{Hypothesis}

PLMI equal to or more than 15 movements per $1 \mathrm{~h}$ of sleep is a predictor of small vessel disease progression.

Null hypothesis: the presence of PLMS with index $\geq 15 / \mathrm{h}$ has no influence on cSVD course.

Alternative hypothesis (one sided): the presence of PLMS with index $\geq 15 / \mathrm{h}$ worsens cSVD course.

\section{The Aim and Objectives}

The aim of the study was to define the role of PLMS in cSVD progression and related cognitive decline progression.

The main objectives of the study are:

1) Assessment of the cognitive function in small vessel disease patients at baseline and in the 2-year follow-up.

2) Assessment of MRI markers of cSVD at baseline and in the 2-year follow-up. 
3) Assessment of sleep quality and health-related quality of life in patients with PLMS in comparison with those without PLMS.

4) Evaluation of PLMS impact on CSVD progression through constructing multiple regression model.

5) Assessment of the association between PLMS and poor sleep quality based on questionnaire data.

\section{METHODS AND DESIGN}

\section{Protocol Outline}

This study was designed as a prospective observational study. It will be conducted in Moscow, Russia (Department of Neurology, I.M. Sechenov First Moscow State Medical University).

In-patients aged 60-75 diagnosed with cSVD according to current diagnostic criteria and neuroimaging standards $(51,54)$ will be evaluated for MRI markers of CSVD and cognitive function over a period of 2 years. General assessment of risk factors for cardiovascular disease will include identification of hypertension (office measured off medication conventional blood pressure of at least $140 / 90 \mathrm{mmHg}$ ), measuring total cholesterol level, calculation of body mass index, as well as revealing by self-report and exploring the medical history the presence of ischemic heart disease, diabetes, and heart rhythm disturbances. Additionally, the duration of smoking and habitual alcohol consumption will be assessed. For the latter purpose, a validated Russian version of the Alcohol Use Disorders Identification Test (AUDIT) will be applied (58).

The design of the study is schematically depicted in Figure 1. All enrolled patients will undergo overnight PSG at baseline. Based on PLMI, all patients will be divided into the experimental $(\mathrm{PLMI} \geq 15 / \mathrm{h})$ and control groups (PLMI $<15 / \mathrm{h})$. The assessment of the night sleep and quality of daytime functioning will be made at baseline only. Assessment of cognitive function and brain MRI will take place at weeks 0 (baseline) and 104 (2-year follow-up). The details on the applied study techniques and evaluation of night sleep, cognitive function, and MRI are given in parts 2.4, 2.5 , and 2.6, respectively. The examination protocol is resumed in Table 1.

\section{Participants}

Patients diagnosed with cSVD will be recruited among inhospital patients (1st and 2nd Department of Neurology, University Clinic 3, Sechenov First Moscow State Medical University, Moscow). According to the Standards for Reporting Vascular Changes on Neuroimaging released in 2013, the neuroimaging characteristics of cSVD consist of WMH, recent small subcortical infarct, cerebral microbleeds, perivascular space, lacune of presumed vascular origin, and brain atrophy (4). The brain MRI of the patient will be assessed by trained radiologist.

Potential participants will be screened for eligibility criteria.

\section{Inclusion Criteria}

- Age between 60 and 75 years.

- Diagnosis of a cSVD-made on the basis of MRI and clinical features, according to current neuroimaging standards for research into small vessel disease (4) and current diagnostic criteria (7).

- Ability to follow the procedures of the study, fluent in Russian language-assessed by self-report.

\section{Non-inclusion Criteria}

- History of symptomatic stroke, brain tumor, or traumatic brain injury-assessed by clinical history.

- Hypothyroidism, B12 deficiency, HIV/AIDS, neurosyphilis, autoimmune, and connective tissue diseases-assessed by clinical history.

\section{Exclusion Criteria}

- History of psychiatric comorbidities and substance use disorder-assessed by self-report, clinical history, and the AUDIT.

- Significant vision loss (leading to difficulties with performing cognitive tasks) — assessed by self-report and clinical history.

- Presence of dementia-assessed by history and clinical examination.

- Movement disorders (e.g., tremor, dystonia) that slow down the speed of performing cognitive tasks-assessed by clinical examination.

- Obstructive sleep apnea syndrome-according to the criteria of International Classification of Sleep Disorders III (14).

- Restless legs syndrome-according to the criteria of the International Restless Legs Syndrome Study Group (63).

- Current intake of neuroleptics, benzodiazepines, selective serotonin reuptake inhibitors, and hypnotics-assessed by self-report and clinical history.

Before recruitment, the MDs will explain to each participant the aim and nature of the study, the main steps to be undertaken, the expected duration, the potential risks and benefits and any discomfort it may lead to, voluntary participation, withdrawal from the study at any time, and that withdrawal of consent will not affect his or her subsequent medical assistance and treatment. After eligibility assessment, potential participants will receive an information paper and a consent form. The consent form will be signed before any study procedures begin. The informed consent (IC) is compiled in accordance with the National Standard of the Russian Federation for Clinical investigations and good clinical practice (GOST R ISO 14155-2014) for the creation of IC for information transfer (64). The protocol of the study and the content of the IC have been approved by the local ethics committee of the I.M. Sechenov Moscow Medical University (no. 15-19/25.11.2019). The study has been registered in ClinicalTrials.gov Protocol Registration and Results System (Identifier: NCT04569643 29.09.2020). The scheduled time for patient recruitment is September 2021 to September 2024. This period will be extended if necessary.

The patients responding to the criteria of inclusion without conditions listed in part 2.2.2 will undergo the routine general clinical assessment and study procedures (nocturnal PSG, neuropsychological assessment, and brain MRI). Patients with identified apnea/hypopnea index (AHI) equal to or more than 5 will be excluded, as well as subjects with conditions mentioned 


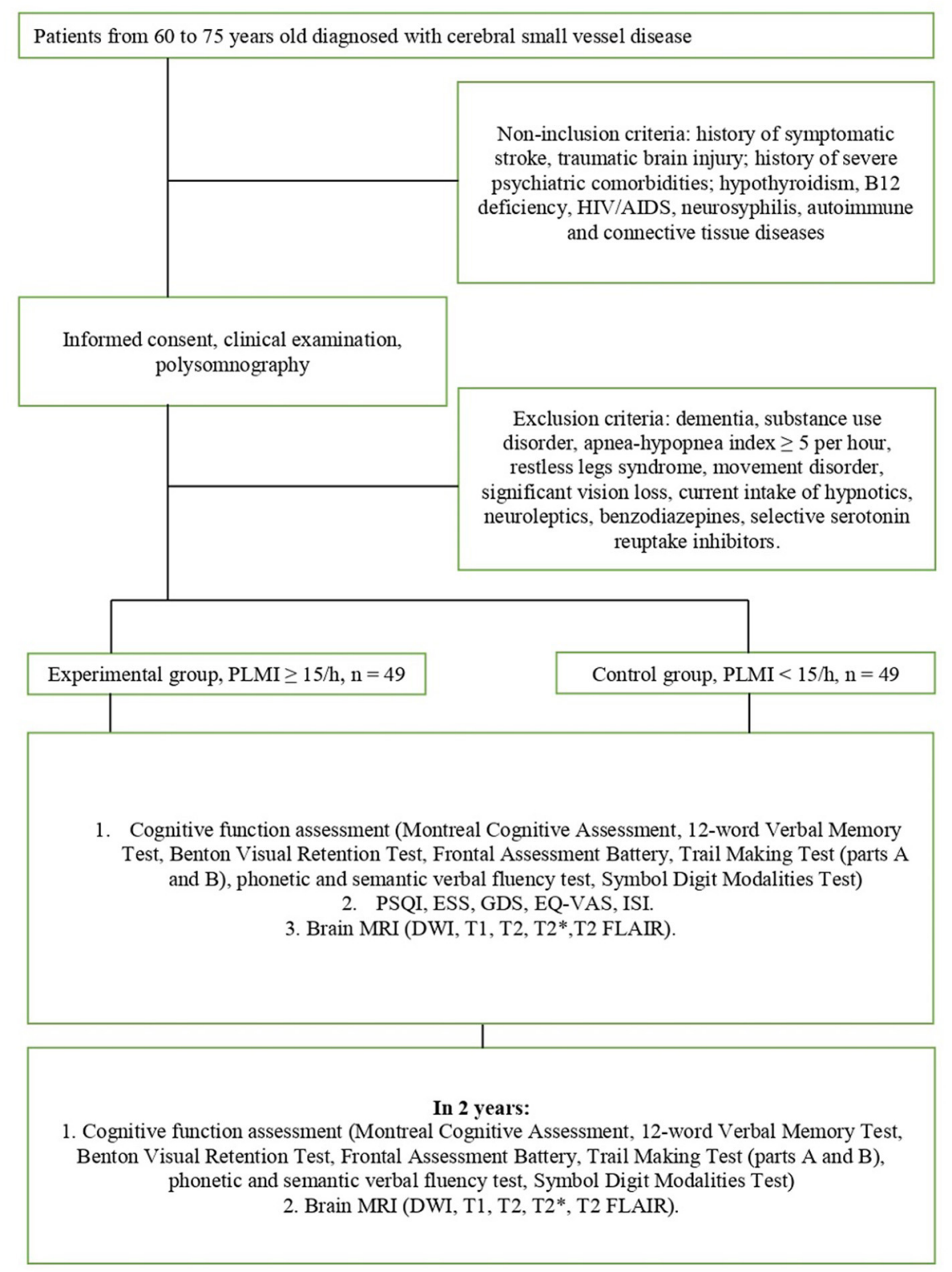

FIGURE 1 | Design of the study.

in part 2.2.3. All enrolled patients will be given recommendation according to 2019 American College of Cardiology/American Heart Association Guideline on the Primary Prevention of Cardiovascular Disease (65) for the purpose to manage generally recognized risk factors for cardiovascular disease.

\section{Nocturnal PSG}

Participants will undergo nocturnal stationary PSG (SOMNOscreen $^{\mathrm{TM}}$, SOMNOmedics GmbH, Germany) at baseline. The PSG protocol will include electroencephalography (EEG) with registration of six monopolar recordings (Fp1A2, Fp2A1, C3A2, C4A1, O1A2, and O2A1); two electrooculography
(EOG) channels; one electromyography (EMG) channel for chin muscles; two EMG channels for right and left tibialis anterior muscles; electrocardiography (ECG); recording of chest and abdominal movements, respiratory flow signal, blood oxygen saturation, and snoring; and registration of body position. Synchronized video recording will be made. Trained somnologist will analyze the data manually in accordance with criteria of The American Association of Sleep Medicine Manual for the scoring the sleep and associated events, version 2.6 (2020) (66). SOMNOwatch software "DOMINO ${ }^{\circledR}$ version 2.9.0" (SOMNOmedics GmbH, Germany) will be used for processing of records. 
TABLE 1 | Examination protocol.

\begin{tabular}{|c|c|c|c|c|}
\hline Methods & Baseline & 2-years follow-up & Unit of measurement & Range \\
\hline \multirow[t]{10}{*}{ Polysomnography } & TST & None & Min & Not defined \\
\hline & SOL & None & Min & Not defined \\
\hline & WASO & None & Min & Not defined \\
\hline & SE & None & $\%$ & $0-100$ \\
\hline & $\mathrm{AHI}$ & None & Number per hour & Not defined \\
\hline & PLMI & None & Number per hour & Not defined \\
\hline & $\mathrm{Pl}$ & None & None & $0-1$ \\
\hline & SILMS index & None & Number per hour & Not defined \\
\hline & PLMWI & None & Number per hour & Not defined \\
\hline & PLMAl & None & Number per hour & Not defined \\
\hline & GDS & None & Points & $0-30$ \\
\hline & EQ-VAS & None & Points & $0-100$ \\
\hline & $|S|$ & None & Points & $0-28$ \\
\hline \multirow[t]{8}{*}{ Cognitive assessment } & MoCA score & MoCA score, percentage change & Points, \% & $0-30$ \\
\hline & 12 word test total score & 12 word test total score, percentage change & Points, \% & $0-24$ \\
\hline & BVRT score & BVRT score, percentage change & Points, \% & $0-10$ \\
\hline & FAB score & FAB score, percentage change & Points, \% & $0-18$ \\
\hline & TMT-A time & TMT-A time, percentage change & $\mathrm{sec}, \%$ & Not defined \\
\hline & TMT-B time & TMT-B time, percentage change & sec, \% & Not defined \\
\hline & $\mathrm{B} / \mathrm{A}$ ratio & $\mathrm{B} / \mathrm{A}$ ratio, percentage change & None, \% & Not defined \\
\hline & SDMT score & SDMT score, percentage change & points, $\%$ & not defined \\
\hline
\end{tabular}

BVRT, Benton Visual Retention Test; B/A ratio, TMT B time divided to TMT A time; PVFT, Phonemic Verbal Fluency Test; SVFT, Semantic Verbal Fluency Test.

Sleep parameters will be calculated taking into account rules for recording and scoring periodic leg movements in sleep and wakefulness determined by the International Restless Legs Syndrome Study Group in 2006 (67) and then revised in 2016 (68). Following values will be obtained: total sleep time (TST), sleep-onset latency (SOL), wake after sleep onset (WASO), sleep efficiency (SE; percentage TST of time in bed), AHI, total number of LMs, number of LM per hour of sleep LM index (LMI), PLMI, index of periodic limb movements with arousals within $3 \mathrm{~s}$ of movement termination (PLMAI), periodic limb movements of wakefulness index (PLMWI), periodicity index (PI), and shortinterval leg movements index (SILMS index).

Patients with obstructive AHI (equal to or more than five episodes per hour of sleep) will be excluded from the study and will be given recommendations for following examination and treatment.
Movements with a duration equal to or more than $0.5 \mathrm{~s}$ are regarded as leg movements during the sleep (LMs), and those with a duration of $<10 \mathrm{~s}$ are candidate LMs (68). Two to four unilateral candidate LMs from the two legs overlapping each other within 0.5-s interval are referred to as one bilateral candidate LM if the total duration is $<15 \mathrm{~s}$. The PLMS are defined as candidate LMs series of four or more, separated by more than 5 and $<90$ s and not interrupted by LM $>10$ s $(68,69)$. The total number of LMs during the sleep will be divided by TST to obtain LMI. The PLMI will be calculated as the number of PLMS per hour of sleep (70). Periodic limb movements during wakefulness (PLMW) are movements that meet the criteria of PLMS but occur during bedtime when patient is awake. PLMAI is a number of associations of PLMS series with EEG arousals per hour of sleep (71). The number of PLMW per hour of wakefulness during bedtime is PLMW index. The number of intermovement 
intervals that will be 10-90 s long and all in sequences of at least three will be divided by the total number of intervals to calculate the PI $(70,72)$. This index lies within 0 (absence of periodicity) to 1 (complete periodicity). Total number of doublets of LMs with intermovement interval $<10 \mathrm{~s}$ is divided by TST to gain SILMS index (73). Experimental and control groups will be formed based on PLMI. The experimental group will include patients with PLMI $\geq 15 / h$, and the control group will include subjects with PLMI $<15 /$ h.

\section{Questionnaires}

Self-reporting scales and questionnaires will be used to assess sleep quality, daytime functioning, and mood. The Pittsburgh Sleep Quality Index (PSQI) (74) is a self-report questionnaire that estimates sleep quality over a 1-month time interval. It includes seven components that assess different aspects of sleep quality, and each of components has a range of $0-3$ points. The Global PSQI Score is the sum of the seven component scores, and it has a range of $0-21$ points, where " 0 " reflects no difficulty and " 21 " reflects severe difficulties in all areas. The Russian version was approved by authors (75) and estimated in the Russian population (76).

The Insomnia Severity Index (ISI) is a brief self-reported tool measuring the perception of the subject of both nocturnal and diurnal symptoms of insomnia (77); the total score ranges from "0" (no symptoms) to " 28 " (severe symptoms). There is a Ph.D. research made by Rasskazova E.I. that contains a validation of the Russian version of the ISI (78).

The Epworth Sleepiness Scale (ESS) is a questionnaire that measures the subjective degree of sleepiness in common life situations. The patient is told to assess his or her chance to fall asleep in each of eight situations with a 4-point scale. The score of the ESS is the sum of these points; it ranges from 4 to 24 (79). The Russian version of the ESS was approved by the authors (80).

The Visual Analog Scale of Quality of Life (EQ-VAS), the part of EQ-5D, provides information about the health-related quality of life self-assessment of the patient (81). The Russian version of the EQ-5D is validated (82). The visual analog scale has 100 grades, where " 100 " represents the best imaginable health state, and " 0 " represents the worst imaginable health state (83). Geriatric Depression Scale (GDS) will be used for mood disorders assessment. It is a 30-item, self-report instrument that uses a "yes/no" format (84). GDS is useful for assessing patients with mild and moderate cognitive impairments, and its score ranges from 0 to 30; a greater score represents a greater risk of depression. The Russian version of the GDS is available and included in the Guidelines for Cognitive Impairment in Older adults approved by the Ministry of Health of the Russian Federation (85).

\section{Assessment of Cognitive Function}

According to the "Diagnostic and Statistical Manual of Mental Disorders, Fifth Edition" (DSM-5), there are six domains of cognitive function: executive function, learning and memory, perceptual motor function, language, complex attention, and social cognition (86). Each domain has subdomains. Executive function consists of working memory, planning, inhibitory control, responding to feedback, flexibility, and decision making. Learning and memory include semantic and autobiographical long-term memory, free and clued recall, recognition memory, and implicit learning. Perceptual motor function is divided into visual perception, visuoconstructional reasoning, and perceptual motor coordination. Language as a cognitive domain includes the ability of grammar and syntax, object naming, fluency, word finding, and receptive language. Complex attention consists of sustained, divided and selective attention, and processing speed. Finally, social cognition includes recognition of emotions and theory of mind. Each domain except social cognition will be evaluated at baseline and after 2 years of follow-up. Both patient groups will receive identical test protocols.

Global cognition including learning and memory visual perception, visuoconstructional reasoning, executive functions, language, and complex attention will be assessed with the validated Russian version of the Montreal Cognitive Assessment (MoCA); the score ranges from 0 to $30(87,88)$. The Russian translation of the MoCA version 7.1 will be used (89).

For evaluation of learning and memory, the 12-word Verbal Memory Test and the Benton Visual Retention Test will be performed. The 12-word verbal memory test measures immediate and delayed recall of verbal material (a list of 12 nouns) using multiple list learning trials with a reminding paradigm (90). The score is the sum of immediate and delayed recall, and it ranges from 0 to 24 . The Benton Visual Retention Test measures recall for 10 images with geometric designs; the first two images consist of one major geometric figure, and the other eight images are composed of two major geometric figures and a smaller peripheral figure (91). A multiple-choice administration will be used, when each image is showed for $10 \mathrm{~s}$ and then withdrawn; immediately after exposure, the patient is shown a multiple-choice card with four similar images. The subject should choose the identical one to the stimulus design image. The score of the Benton Test is the number of correctly recalled designs, and it ranges from 0 to 10 .

The executive function domain will be measured with Frontal Assessment Battery (FAB) (92) ranging from 0 to 18, Trail Making Test (TMT) (93), parts A (the subject should draw a line linking consecutive numbers from 1 to 25 ) and B (the subject should draw a line linking alternating numbers and letters in sequence). Part B consists of numbers from 1 to 12 and the first 12 letters of the Russian alphabet. The time to complete each part of TMT is recorded, and the examiner will point out errors as they occur. The score is the time in seconds required for performing each part, and A/B ratio that reflects set shifting ability will be also estimated (94). The executive function is also estimated by phonetic verbal fluency test (naming nouns starting with a defined letter in $1 \mathrm{~min}$ ), which is included in MoCA. The Russian version of the FAB is included in Guidelines for Cognitive Impairment in Older Adults approved by the Ministry of Health of the Russian Federation (85).

Complex attention and processing speed will be measured with the Symbol Digit Modalities Test (SDMT) (95), which is a subtest of the Wechsler Adult Intelligence Scale-Revised (WAISR) (96). The score is the correct number of substitutions in $90 \mathrm{s.}$ Only the written response format of the SDMT will be conducted. 
Language will be assessed with animal naming against the clock in $1 \mathrm{~min}$ (Semantic Verbal Fluency Test) that measures the ability to rapidly generate words from a specified category (97). The score of the task is the number of animals named.

\section{Neuroimaging}

All subjects will undergo brain MRI scanning at baseline as a routine procedure and in the 2-year follow-up as a study procedure. The imaging protocol will include axial T1, T2, T2*, fluid attenuated inversion recovery (FLAIR), and diffusion weighted imaging (DWI) scans that will be made on a 3-Tesla scanner (Siemens, Erlangen, Germany). Slice thickness will be $5 \mathrm{~mm}$, with an interslice gap of $1 \mathrm{~mm}$ and a matrix size of 192 $\times 256$ pixels.

All the images will be processed with volBrain online software pipeline (98) to make an automated volumetric measurement of WMH. The volume of the white matter lesion will be calculated separately for the periventricular, juxtacortical, infratentorial, and deep white matter areas as well as the total volume of the lesion. Baseline volumes will be subtracted from follow-up volumes to obtain absolute $\mathrm{WMH}$ volume change. In addition, proportional volume change will be calculated by dividing the absolute $\mathrm{WMH}$ volume change by baseline $\mathrm{WMH}$ volume with a percentage display of results.

One trained and blinded rater will evaluate MRI scans with three cross-sectional visual scales [the Fazekas Scale (99), the Scheltens Scale (100), and the cSVD Score (101)] and the Rotterdam progression scale (102). The Fazekas Scale has a range of $0-6$. Both in the periventricular and in the subcortical regions, scores $0-3$ can be given for absence, mild, moderate, or severe lesions, respectively (99). The Scheltens Rating Scale ranges from 0 to 84: scores $0-6$ can be given in 13 subcortical regions (frontal, parietal, temporal and occipital lobes, caudate, putamen, globus pallidus, thalamus, internal capsule, cerebellum, mesencephalon, pons, and medulla) and scores $0-2$ for three periventricular regions (frontal caps, occipital caps, and lateral ventricles bands) (100). Change scores will be obtained by subtracting the baseline scores from the follow-up scores. Calculating the total SVD score (range 0-4) will be made by adding up of scores for each cSVD marker: 1 point for presence of any lacunes, 1 point for one or more cerebral microbleeds, 1 point for presence of enlarged perivascular spaces (PVS) in the basal ganglia, and 1 point for presence of moderate to severe PVS in other regions (101). The Rotterdam Progression Scale ranges from -7 to +7 , measuring changes in $\mathrm{WMH}(-1$, decrease; 0 , no change; and +1 , increase) for three periventricular regions (frontal caps, lateral bands, and occipital caps) and four subcortical regions (frontal, parietal, temporal, and occipital) (102).

\section{Outcomes}

The primary outcome variable is total $\mathrm{WMH}$ volume change in percentage from baseline. The minimal clinically important change would be calculated based on baseline standard deviation as it was suggested by Lemieux et al. (103): the smallest effect size will be calculated by multiplying the baseline standard deviation by 0.2 .

The secondary outcomes are the WMH volume change in each area of interests (periventricular, juxtacortical, infratentorial, and deep white matter), change in cognitive performance (results of each test will be processed separately) in percentage from baseline, cerebral infarct incidence, non-traumatic intracranial hemorrhage incidence, transient ischemic attack incidence, and rate of mortality due to stroke.

\section{Statistical Analysis and Sample Size Calculation}

The baseline values of MRI scale scores and cognitive tests (listed in Table 1) will be subtracted from the corresponding follow-up values, and percent change from baseline will be calculated. Based on the PLMI ( $\geq 15 / \mathrm{h}$ or $<15 / \mathrm{h}$ ), two groups will be formed. In order to reproduce results of the cross-sectional study conducted by Kang et al. (51), group comparisons will be made using the Mann-Whitney test for non-parametric statistics or unpaired $t$ test, depending on whether the distribution is normal. To assess the association between the PLMI and total cerebral SVD sum score, as well as WMH volume, Fazekas Scale score, and Scheltens Scale separately, Spearman correlation analysis will be applied. To confirm the data obtained by Leng et al. (54) and complement it with a more detailed cognitive assessment, logistic regression will be utilized to study the association between PLMS and clinically significant changes in cognition from the baseline to the follow-up visit.

Multiple regression analysis will be used to estimate the capability of each of the characteristics of PLMS (LMs, LMI, PLMI, PI, and SILMS index) to predict each of the outcome variables (except for stroke incidence and mortality rate). Adjustment for main predictors of cardiovascular risk (age, hypertension level, body mass index, diabetes mellitus, ischemic heart disease, cholesterol level, and smoking) will be made to determine if the associations are independent of these factors. Comparison of stroke incidence (separately for cerebral infarct, non-traumatic intracranial hemorrhage, and transient ischemic attack) as well as mortality rate due to stroke between two groups will be made using Pearson's chi-square test.

Spearman correlation test will be applied to evaluate the association between the PLMI and scores of self-reported questionnaires (listed in Table 1) and assess the influence of PLMS on sleep quality, mood, and daytime functioning. The level of statistical significance will be set at $p<0.05$. All statistical analyses will be performed using SPSS version 21.0 (SPSS Inc., Chicago, IL, USA).

Since the progression of cSVD features depending on PLMI is the key point of our study and linear regression will be applied, we calculated the sample size $(\mathrm{N})$ based on this type of design. As mentioned above, there are seven independent variables ( $p$ $=7$ ): age, arterial hypertension level, body mass index, blood sugar level, ischemic heart disease, cholesterol level, and smoking duration, and one tested parameter (PLMI or other parameters of PLMS). The sample size should be calculated depending on effect size: $\mathrm{N}=392+\mathrm{p}$ for small, $\mathrm{N}=52+\mathrm{p}$ for medium, and $\mathrm{N}=22+\mathrm{p}$ for large effect size respectively (104). Thus, for revealing medium effect size, $\mathrm{N}=52+7=59$ participants should be enrolled, and for revealing small effect size, $\mathrm{N}=$ $392+$ p, or $392+7=399$ participants should be enrolled. A large effect size of PLMS is not expected. Therefore, 59 patients should be enrolled initially, and when no effect is revealed, the 
sample size should be enlarged up to 399 . On the other hand, a comparison of cSVD feature prevalence between two groups is also planned as interim analysis. For this purpose, unpaired $t$-test (or Mann-Whitney test, depending on whether the distribution is normal) will be applied, and it requires standard deviation (SD) to calculate the sample size. When SD $=1.22$ [obtained by Kang et al. (51)] and given probability for rejecting the null hypothesis $\alpha=0.05$, probability of failing to reject the null hypothesis under the alternative hypothesis $\beta=0.2$ and effect size $=0.5$, group size $N=49$ is received when rounded up to the next highest integer. Therefore, at least $49^{*} 2=98$ patients should be enrolled, but this number could be changed after another value of SD will be received.

\section{DISCUSSION}

PLMS are regarded as the possible sign of repetitive autonomic dysfunction during night sleep. Nevertheless, it is not known if this autonomic dysfunction can worsen the course of cardiovascular pathology, as well as increase the risk of cardiovascular events. In this regard, the investigation of the long-term effects of PLMS seems to be worth researching.

The proposed protocol of the study allows estimate influence of PLMS on both the course of CSVD and incidence of stroke. WMH increase and cognitive decline, which are the key features of cSVD progression, will be assessed. In comparison with the researches conducted by Kang et al. (51) and Del Brutto et al. (105), our study has a longitudinal observational design, which seems to be more appropriate for the purpose of risk factors identification than a cross-sectional design. These researchers came to the opposite conclusions in their studies that seem to support the utility of further investigation of the problem. Besides, the proposed protocol suggests a more detailed MRI assessment. Estimation of different brain regions separately is another strength of our study.

In comparison with another prototype study conducted by Leng et al. (54), the proposed study has also some strengths. The neuropsychological examination is more detailed and comprehensive. Particular attention is paid to the assessment of executive functions since they are affected by vascular cognitive impairment to a greater extent.

The results of the proposed research have potential practical value because PLMS is a treatable condition. If PLMS is identified as a predictor for cSVD progression, it could be regarded as a modifiable risk factor for cSVD, which can be managed. Eventually, it may contribute to improving patient outcomes.

Several limitations of the study should be considered. The design of the study does not allow to reveal the exact mechanism of how PLMS leads to cSVD progression and cognitive decline. If the hypothesis of the research is confirmed, we will be able to assume the possible underlying process only. Finally, because of the short follow-up period, no differences between groups might be yielded and the role of PLMS can be underestimated.

The reported association between high PLMI and transient sympathetic increase does not clarify whether it is a causeand-effect connection or these events have the same origin. In this regard, more research using controlled trials is needed to demonstrate the positive impact of prolonged treatment of PLMS on cardiovascular health. The absence of a treated group is also one of the limitations of our study. It could establish the utility of pharmacological reduction PLMS.

Another significant limitation of the proposed study design should be discussed. The baseline level of both cognitive function and $\mathrm{WMH}$ volume determinates the speed of further progression of the cSVD. Therefore, the difference between groups at baseline will be the reason for the difference in disease progression. In order to deal with this problem, multiple regression analysis is applied and the baseline level will be included in multiple regression equation. Nevertheless, there is a potential bias related to the eligibility criteria. Only a subgroup of patients with AHI under 5 per hour will be included. Taking into account that the large proportion of cSVD patients has at least mild SOAS, the research will encompass only a small part of cSVD patients. However, we have to exclude even mild SOAS. Obstructive sleep apnea syndrome is accompanied by chronic sleep fragmentation as well as chronic hypoxemia that could worsen cognitive performance. Although the impact of mild SOAS on cognitive performance is still controversial (106-108), we cannot be sure that the presence of this condition will not influence the outcome. Taking into account that LMs in general and PLMS, in particular, could be associated with respiratory events, we would not include patients with even mild OSA with the purpose to avoid the presence of confounding factors.

Notwithstanding these limitations, the results of our study could elucidate the role of PLMS in cSVD progression and provide information about its possible influence on assessed outcomes-cognitive decline progression and MRI changes.

\section{DISSEMINATION}

Results of this trial will be disseminated via peer-reviewed journal publications. Primary endpoint results will be reported in a single publication. Other findings will be published separately. The results of this study will also be available on the ClinicalTrials.gov website.

\section{AUTHOR CONTRIBUTIONS}

ES, MP, and IF developed the study protocol and contributed to its description. ES and MP contributed to the eligibility criteria of the study and elaboration of the neuropsychology assessment. IF contributed to the sample size calculation and to the plan of statistical processing and defined details of PLMS assessment. All authors contributed to the conception and design of the study, article, and approved the submitted version.

\section{FUNDING}

The study is supported by the scholarship program of German Academic Exchange Service (DAAD), funding program "Binationally Supervised Doctoral Degrees/Cotutelle", personal ref. no. 91775226 , ID 57507869. 


\section{REFERENCES}

1. Pantoni L. Cerebral small vessel disease: from pathogenesis and clinical characteristics to therapeutic challenges. Lancet Neurol. (2010) 9:689701. doi: 10.1016/S1474-4422(10)70104-6

2. Prins ND, Scheltens $P$. White matter hyperintensities, cognitive impairment and dementia: an update. Nat Rev Neurol. (2015) 11:157-65. doi: 10.1038/nrneurol.2015.10

3. Gorelick PB, Scuteri A, Black SE, Decarli C, Greenberg SM, Iadecola C, et al. Vascular contributions to cognitive impairment and dementia: a statement for healthcare professionals from the american heart association/american stroke association. Stroke. (2011) 42:2672-713. doi: 10.1161/STR.0b013e3182299496

4. Wardlaw JM, Smith EE, Biessels GJ, Cordonnier C, Fazekas F, Frayne R, et al. Neuroimaging standards for research into small vessel disease and its contribution to ageing and neurodegeneration. Lancet Neurol. (2013) 12:822-38. doi: 10.1016/S1474-4422(13)70124-8

5. Debette S, Markus HS. The clinical importance of white matter hyperintensities on brain magnetic resonance imaging: systematic review and meta-analysis. BMJ. (2010) 341:c3666. doi: 10.1136/bmj.c3666

6. van Leijsen EMC, de Leeuw F-E, Tuladhar AM. Disease progression and regression in sporadic small vessel disease-insights from neuroimaging. Clin Sci. (2017) 131:1191-206. doi: 10.1042/CS20160384

7. Rosenberg GA, Wallin A, Wardlaw JM, Markus HS, Montaner J, Wolfson L, et al. Consensus statement for diagnosis of subcortical small vessel disease. $J$ Cereb Blood Flow Metab. (2016) 36:6-25. doi: 10.1038/jcbfm.2015.172

8. Mims KN, Kirsch D. Sleep and stroke. Sleep Med Clin. (2016) 11:3951. doi: 10.1016/j.jsmc.2015.10.009

9. Culebras A. Sleep stroke and poststroke. Neurol Clin. (2012) 30:127584. doi: 10.1016/j.ncl.2012.08.017

10. Koo BB, Sillau S, Dean DA, Lutsey PL, Redline S. Periodic limb movements during sleep and prevalent hypertension in the multi-ethnic study of atherosclerosis. Hypertension. (2015) 65:70-7. doi: 10.1161/HYPERTENSIONAHA.114.04193

11. May AM, Blackwell T, Stone KL, Cawthon PM, Sauer WH, Varosy $\mathrm{PD}$, et al. Longitudinal relationships of periodic limb movements during sleep and incident atrial fibrillation. Sleep Med. (2017) 25:7886. doi: 10.1016/j.sleep.2016.08.009

12. Yumino D, Wang H, Floras JS, Newton GE, Mak S, Ruttanaumpawan $\mathrm{P}$, et al. Relation of periodic leg movements during sleep and mortality in patients with systolic heart failure. Am J Cardiol. (2011) 107:44751. doi: 10.1016/j.amjcard.2010.09.037

13. Stefani A, Högl B. Diagnostic criteria, differential diagnosis, and treatment of minor motor activity and less well-known movement disorders of sleep. Curr Treat Options Neurol. (2019) 21:1. doi: 10.1007/s11940-019-0543-8

14. Sateia MJ. International classification of sleep disorders-third edition. Chest. (2014) 146:1387-94. doi: 10.1378/chest.14-0970

15. Poluektov MG. Somnology and Sleep Medicine. National Guide (in Russian). Moscow: MedForum (2016).

16. Hornyak M, Feige B, Riemann D, Voderholzer U. Periodic leg movements in sleep and periodic limb movement disorder: prevalence, clinical significance and treatment. Sleep Med Rev. (2006) 10:169-77. doi: 10.1016/j.smrv.2005.12.003

17. Hanly PJ, Zuberi-Khokhar N. Periodic limb movements during sleep in patients with congestive heart failure. Chest. (1996) 109:1497502. doi: 10.1378/chest.109.6.1497

18. Gann H, Feige B, Fasihi S, van Calker D, Voderholzer U, Riemann D. Periodic limb movements during sleep in alcohol dependent patients. Eur Arch Psychiatry Clin Neurosci. (2002) 252:124-9. doi: 10.1007/s00406-002-0371-8

19. Voderholzer U, Müller N, Haag C, Riemann D, Straube A. Periodic limb movements during sleep are a frequent finding in patients with Gilles de la Tourette's syndrome. J Neurol. (1997) 244:5216. doi: 10.1007/s004150050136

20. Nogués M, Cammarota A, Leiguarda R, Rivero A, Pardal A, Encabo H. Periodic limb movements in syringomyelia and syringobulbia. Mov Disord. (2000) 15:113-9. doi: 10.1002/1531-8257(200001)15:1\&lt;113::AIDMDS1017\&gt;3.0.CO;2-Y
21. Wetter TC, Collado-Seidel V, Pollmächer T, Yassouridis A, Trenkwalder C. Sleep and periodic leg movement patterns in drug-free patients with Parkinson's disease and multiple system atrophy. Sleep. (2000) 23:3617. doi: $10.1093 /$ sleep/23.3.1c

22. Fulda S. The role of periodic limb movements during sleep in restless legs syndrome. Sleep Med Clin. (2015) 10:241-8. doi: 10.1016/j.jsmc.2015.05.013

23. Hening $\mathrm{W}$. The clinical neurophysiology of the restless legs syndrome and periodic limb movements. Part I: diagnosis, assessment, and characterization. Clin Neurophysiol. (2004) 115:1965-74. doi: 10.1016/j.clinph.2004.03.032

24. Lugaresi E, Coccagna G, Tassinari CA, Ambrosetto C. [Polygraphic data on motor phenomena in the restless legs syndrome]. Riv Neurol. (1965) 35:550-61.

25. Ondo WG, Vuong KD, Jankovic J. Exploring the relationship between Parkinson disease and restless legs syndrome. Arch Neurol. (2002) 59:4214. doi: 10.1001/archneur.59.3.421

26. Trenkwalder C, Collado Seidel V, Kazenwadel J, Wetter TC, Oertel W, Selzer $\mathrm{R}$, et al. One-year treatment with standard and sustained-release levodopa: appropriate long-term treatment of restless legs syndrome? Mov Disord. (2003) 18:1184-9. doi: 10.1002/mds.10503

27. Manconi M, Ferri R, Zucconi M, Bassetti CL, Fulda S, Aricò D, et al. Dissociation of periodic leg movements from arousals in restless legs syndrome. Ann Neurol. (2012) 71:834-44. doi: 10.1002/ana.23565

28. Rijsman RM, Stam CJ, Weerd AW de. Abnormal H-reflexes in periodic limb movement disorder; impact on understanding the pathophysiology of the disorder. Clin Neurophysiol. (2005) 116:204-10. doi: 10.1016/j.clinph.2004.07.022

29. Manconi M, Ferri R, Zucconi M, Clemens S, Rundo F, Oldani A, et al. Effects of acute dopamine-agonist treatment in restless legs syndrome on heart rate variability during sleep. Sleep Med. (2011) 12:4755. doi: 10.1016/j.sleep.2010.03.019

30. Barraud Q, Obeid I, Aubert I, Barrière G, Contamin H, McGuire $S$, et al. Neuroanatomical study of the A11 diencephalospinal pathway in the non-human primate. PLoS ONE. (2010) 5:e13306. doi: 10.1371/journal.pone.0013306

31. Ondo WG, He Y, Rajasekaran S, Le WD. Clinical correlates of 6hydroxydopamine injections into A11 dopaminergic neurons in rats: a possible model for restless legs syndrome. Mov Disord. (2000) 15:15458. doi: 10.1002/1531-8257(200001)15:1\&lt;154::AID-MDS1025\&gt;3.0. $\mathrm{CO} ; 2-\mathrm{Q}$

32. Telles SCL, Alves RC, Chadi G. Periodic limb movements during sleep and restless legs syndrome in patients with ASIA A spinal cord injury. J Neurol Sci. (2011) 303:119-23. doi: 10.1016/j.jns.2010.12.019

33. Salminen AV, Manconi M, Rimpilä V, Luoto TM, Koskinen E, Ferri R, et al. Disconnection between periodic leg movements and cortical arousals in spinal cord injury. J Clin Sleep Med. (2013) 9:1207-9. doi: 10.5664/jcsm.3174

34. Barone DA, Ebben MR, DeGrazia M, Mortara D, Krieger AC. Heart rate variability in restless legs syndrome and periodic limb movements of Sleep. Sleep Sci. (2017) 10:80-6. doi: 10.5935/1984-0063.20170015

35. Sforza E, Pichot V, Barthelemy JC, Haba-Rubio J, Roche F. Cardiovascular variability during periodic leg movements: a spectral analysis approach. Clin Neurophysiol. (2005) 116:1096-104. doi: 10.1016/j.clinph.2004.12.018

36. Pennestri M-H, Montplaisir J, Fradette L, Lavigne G, Colombo R, Lanfranchi PA. Blood pressure changes associated with periodic leg movements during sleep in healthy subjects. Sleep Med. (2013) 14:55561. doi: 10.1016/j.sleep.2013.02.005

37. Palma J-A, Alegre M, Valencia M, Artieda J, Iriarte J, Urrestarazu E. Basal cardiac autonomic tone is normal in patients with periodic leg movements during sleep. J Neural Transm. (2014) 121:385-90. doi: 10.1007/s00702-013-1116-8

38. Sforza E, Roche F, Pichot V. Determinants of nocturnal cardiovascular variability and heart rate arousal response in Restless Legs Syndrome (RLS)/Periodic Limb Movements (PLMS). J Clin Med. (2019) 8:1619. doi: $10.3390 / \mathrm{jcm} 8101619$

39. Guggisberg AG, Hess CW, Mathis J. The significance of the sympathetic nervous system in the pathophysiology of periodic leg movements in sleep. Sleep. (2007) 30:755-66. doi: 10.1093/sleep/30.6.755 
40. Iriarte J, Urrestarazu E, Alegre M, Valencia M, Artieda J. Oscillatory cortical changes during periodic limb movements. Sleep. (2004) 27:1493. doi: 10.1093/sleep/27.8.1493

41. Sieminski M, Pyrzowski J, Partinen M. Periodic limb movements in sleep are followed by increases in EEG activity, blood pressure, and heart rate during sleep. Sleep Breath. (2017) 21:497-503. doi: 10.1007/s11325-017-1476-7

42. May AM, May RD, Bena J, Wang L, Monahan K, Stone KL, et al. Individual periodic limb movements with arousal are temporally associated with nonsustained ventricular tachycardia: a case-crossover analysis. Sleep. (2019) 42:zsz165. doi: 10.1093/sleep/zsz067.668

43. Winkelman JW. The evoked heart rate response to periodic leg movements of sleep. Sleep. (1999) 22:575-80. doi: 10.1093/sleep/22.5.575

44. Coelho FMS, Georgsson H, Narayansingh M, Swartz RH, Murray BJ. Higher prevalence of periodic limb movements of sleep in patients with history of stroke. J Clin Sleep Med. (2010) 06:428-30. doi: 10.5664/jcsm.27930

45. Manconi M, Fanfulla F, Ferri R, Miano S, Haba-Rubio J, Heinzer $\mathrm{R}$, et al. Periodic limb movements during sleep in stroke/TIA: prevalence, course, and cardiovascular burden. Neurology. (2018) 90:e1663-e72. doi: 10.1212/WNL.0000000000005471

46. Innes KE, Selfe TK, Agarwal P. Restless legs syndrome and conditions associated with metabolic dysregulation, sympathoadrenal dysfunction, and cardiovascular disease risk: a systematic review. Sleep Med Rev. (2012) 16:309-39. doi: 10.1016/j.smrv.2011.04.001

47. Sieminski M, Chwojnicki K, Partinen M. Higher nocturnal systolic blood pressure in patients with restless legs syndrome compared with patients with insomnia. Sleep Med. (2017) 32:229-33. doi: 10.1016/j.sleep.2016.07.025

48. Espinar-Sierra J, Vela-Bueno A, Luque-Otero M. Periodic leg movements in sleep in essential hypertension. Psychiatry Clin Neurosci. (1997) 51:1037. doi: 10.1111/j.1440-1819.1997.tb02370.x

49. Kendzerska T, Kamra M, Murray BJ, Boulos MI. Incident cardiovascular events and death in individuals with restless legs syndrome or periodic limb movements in sleep: a systematic review/. Sleep. (2017) 40(3). doi: 10.1093/sleep/zsx013

50. Yatsu S, Kasai T, Suda S, Matsumoto H, Shiroshita N, Kato M, et al. Impact on clinical outcomes of periodic leg movements during sleep in hospitalized patients following acute decompensated heart failure. Circ J. (2017) 81:495500. doi: 10.1253/circj.CJ-16-0934

51. Kang MK, Koo DL, Shin JH, Kwon H-M, Nam H. Association between periodic limb movements during sleep and cerebral small vessel disease. Sleep Meda. (2018) 51:47-52. doi: 10.1016/j.sleep.2018.06.018

52. Spektor ED, Poluektov MG. Periodic limb movements in sleep and clinicomorphological features of cerebral small vessel disease. Zh Nevrol Psikhiatr Im S S Korsakova. (2021) 121:75-9. doi: 10.17116/jnevro202112104275

53. Boulos MI, Murray BJ, Muir RT, Gao F, Szilagyi GM, Huroy M, et al. Periodic limb movements and white matter hyperintensities in firstever minor stroke or high-risk transient ischemic attack. Sleep. (2016) 40:zsw080. doi: 10.1093/sleep/zsw080

54. Leng Y, Blackwell T, Stone KL, Hoang TD, Redline S, Yaffe K. Periodic limb movements in sleep are associated with greater cognitive decline in older men without dementia. Sleep. (2016) 39:1807-10. doi: 10.5665/sleep.6158

55. Scullin MK, Fairley JA, Trotti LM, Goldstein FC, Factor SA, Bliwise DL. Sleep correlates of trait executive function and memory in Parkinson's disease. $J$ Parkinsons Dis. (2015) 5:49-54. doi: 10.3233/JPD-140475

56. Lambiase MJ, Gabriel KP, Kuller LH, Matthews KA. Sleep and executive function in older women: the moderating effect of physical activity. $J$ Gerontol A Biol Sci Med Sci. (2014) 69:1170-6. doi: 10.1093/gerona/glu038

57. Wilckens KA, Erickson KI, Wheeler ME. Age-related decline in controlled retrieval: the role of the PFC and sleep. Neural Plast. (2012) 2012:624795. doi: 10.1155/2012/624795

58. Roh JH, Lee J-H. Recent updates on subcortical ischemic vascular dementia. J Stroke. (2014) 16:18. doi: 10.5853/jos.2014.16.1.18

59. Peng D. Clinical practice guideline for cognitive impairment of cerebral small vessel disease. Aging Med. (2019) 2:64-73. doi: 10.1002/agm2.12073

60. van Norden AGW, van den Berg HAC, de Laat KF, Gons RAR, van Dijk EJ, de Leeuw F-E. Frontal and temporal microbleeds are related to cognitive function: the Radboud University Nijmegen Diffusion Tensor and
Magnetic Resonance Cohort (RUN DMC) study. Stroke. (2011) 42:33826. doi: 10.1161/STROKEAHA.111.629634

61. Uiterwijk R, Staals J, Huijts M, de Leeuw PW, Kroon AA, van Oostenbrugge RJ. MRI progression of cerebral small vessel disease and cognitive decline in patients with hypertension. J Hypertension. (2017) 35:126370. doi: 10.1097/HJH.0000000000001294

62. Ballard C, McKeith I, O’Brien J, Kalaria R, Jaros E, Ince P, et al. Neuropathological substrates of dementia and depression in vascular dementia, with a particular focus on cases with small infarct volumes. Dement Geriatr Cogn Disord. (2000) 11:59-65. doi: 10.1159/000017215

63. Walters AS. Toward a better definition of the restless legs syndrome. the international restless legs syndrome study group. Mov Disord. (1995) 10:63442.

64. GOST R ISO 14155-2014. Clinical Trials. Good Clinical Practice. Amended on 01.06.2016 Law reference system "Consultant- Plus." Available online at: http://docs.cntd.ru/document/1200110952 (accessed July 22, 2021)

65. Arnett Donna K, Blumenthal Roger S, Albert Michelle A, Buroker Andrew B, Goldberger Zachary D, Hahn Ellen J, et al. 2019 ACC/AHA guideline on the primary prevention of cardiovascular disease: a report of the American College of Cardiology/American heart association task force on clinical practice guidelines. Circulation. (2019) 140:e596646. doi: 10.1161/CIR.0000000000000725

66. Berry R, Quan S, Abreu A, Bibbs M, DelRosso L, Harding S. The AASM Manual for the Scoring of Sleep and Associated Events: Rules, Terminology and Technical Specifications, version 2.6. Darien, IL: American Academy of Sleep Medicine (2020). Available online at: https://aasm.org/clinical-resources/ scoring-manual/ (accessed July 22, 2021)

67. Zucconi M, Ferri R, Allen R, Baier PC, Bruni O, Chokroverty S, et al. The official World Association of Sleep Medicine (WASM) standards for recording and scoring periodic leg movements in sleep (PLMS) and wakefulness (PLMW) developed in collaboration with a task force from the International Restless Legs Syndrome Study Group (IRLSSG). Sleep Med. (2006) 7:175-83. doi: 10.1016/j.sleep.2006.01.001

68. Ferri R, Fulda S, Allen RP, Zucconi M, Bruni O, Chokroverty S, et al. World Association of Sleep Medicine (WASM) 2016 standards for recording and scoring leg movements in polysomnograms developed by a joint task force from the International and the European Restless Legs Syndrome Study Groups (IRLSSG and EURLSSG). Sleep Med. (2016) 26:8695. doi: 10.1016/j.sleep.2016.10.010

69. Bonnet M, Carley D, Carskadon M, Easton P, Guilleminault C, Harper R, et al. Recording and scoring leg movements. The Atlas Task Force. Sleep. (1993) 16:748-59. doi: 10.1093/sleep/16.8.748

70. Ferri R, Fulda S, Manconi M, Högl B, Ehrmann L, Ferini-Strambi L, et al. Night-to-night variability of periodic leg movements during sleep in restless legs syndrome and periodic limb movement disorder: comparison between the periodicity index and the PLMS index. Sleep Med. (2013) 14:2936. doi: 10.1016/j.sleep.2012.08.014

71. Bonnet M, Carley D, Carskadon M, Easton P, Guilleminault C, Harper $\mathrm{R}$, et al. EEG arousals: scoring rules and examples: a preliminary report from the sleep disorders atlas task force of the american sleep disorders association. Sleep. (1992) 15:173-84. doi: 10.1093/sleep/15. 2.174

72. Ferri R, Zucconi M, Manconi M, Plazzi G, Bruni O, Ferini-Strambi L. New approaches to the study of periodic leg movements during sleep in restless legs syndrome. Sleep. (2006) 29:759-69. doi: 10.1093/sleep/29.12.1587

73. Ferri R, Rundo F, Silvani A, Zucconi M, Aricò D, Bruni O, et al. Short-interval leg movements during sleep entail greater cardiac activation than periodic leg movements during sleep in restless legs syndrome patients. J Sleep Res. (2017) 26:602-5. doi: 10.1111/jsr.12529

74. Buysse DJ, Reynolds CF, Monk TH, Berman SR, Kupfer DJ. The Pittsburgh Sleep Quality Index: a new instrument for psychiatric practice and research. Psychiatry Res. (1989) 28:193-213. doi: 10.1016/0165-1781(89) 90047-4

75. Buysse D, Berman S, Kupfer D, Monk T, Reynolds C. PSQI - Pittsburgh Sleep Quality Index. ePROVIDE Online Support for Clinical Outcome Assessments. Available online at: https://eprovide.mapi-trust.org/instruments/pittsburghsleep-quality-index (accessed July 14, 2021) 
76. Kolomeychuk S, Randler C, Churov A, Borisenkov M. PSQI estimation in the sample of Russian students. Int Online J Educ Sci. (2016) 2016:7984. doi: 10.15345/iojes.2016.05.009

77. Morin CM, Belleville G, Bélanger L, Ivers H. The Insomnia Severity Index: psychometric indicators to detect insomnia cases and evaluate treatment response. Sleep. (2011) 34:601-8. doi: 10.1093/sleep/34.5.601

78. Rasskazova EI. PhD dissertation (in Russian) (2008) Available online at: https://www.dissercat.com/content/narusheniya-psikhologicheskoisamoregulyatsii-pri-nevroticheskoi-insomnii (accessed July 22, 2021)

79. Doneh B. Epworth sleepiness scale. Occup Med. (2015) 65:508. doi: 10.1093/occmed/kqv042

80. Johns M. ESS - Epworth Sleepiness Scale. ePROVIDE Online Support for Clinical Outcome Assessments. Available online at: https://eprovide.mapitrust.org/instruments/epworth-sleepiness-scale (accessed July 14, 2021)

81. Devlin NJ, Brooks R. EQ-5D and the EuroQol group: past, present and future. Appl Health Econ Health Policy. (2017) 15:127-37. doi: 10.1007/s40258-017-0310-5

82. Amirdjanova V, Erdes S. Validation of general questionnaire EuroQol5D (EQ-5D) Russian version. Rheumatol Sci Practice. (2007) 69. doi: 10.14412/1995-4484-2007-691

83. EuroQol Group. EuroQol - a new facility for the measurement of health-related quality of life. Health Policy. (1990) 16:199208. doi: 10.1016/0168-8510(90)90421-9

84. Yesavage JA, Brink TL, Rose TL, Lum O, Huang V, Adey M, et al. Development and validation of a geriatric depression screening scale: a preliminary report. J Psychiatr Res. (1982) 17:37-49. doi: 10.1016/0022-3956(82)90033-4

85. Guidelines for Cognitive Impairment in Older Adults Approved by the Ministry Health of the Russian Federation (in Russian). (2020) Available online at: http://www.consultant.ru/document/cons_doc_LAW_367818/ (accessed July 22, 2021)

86. Simpson JR. DSM-5 and neurocognitive disorders. J Am Acad Psychiatry Law Online. (2014) 42:159-64. PMID. 4986342.

87. Nasreddine ZS, Phillips NA, Bédirian V, Charbonneau S, Whitehead V, Collin I, et al. The Montreal Cognitive Assessment, MoCA: a brief screening tool for mild cognitive impairment. J Am Geriatr Soc. (2005) 53:6959. doi: 10.1111/j.1532-5415.2005.53221.x

88. Freud T, Vostrikov A, Dwolatzky T, Punchik B, Press Y. Validation of the Russian version of the MoCA test as a cognitive screening instrument in cognitively asymptomatic older individuals and those with mild cognitive impairment. Front Med. (2020) 7:447. doi: 10.3389/fmed.2020.00447

89. MoCA Test. MoCA Montreal - Cognitive Assessment. Available online at: https://www.mocatest.org/paper/ (accessed August 16, 2020)

90. Zakharov VV, Voznesenskaya TG. Neuropsychiatric Disorders: Diagnostic Tools (in Russian). Moscow: "MEDpress-Inform" Limited Liability Company (2016).

91. Spreen O, Strauss E, Sherman. A Compendium of Neuropsychological Tests: Administration, Norms, and Commentary, Appl. Neuropsychol. 14:62-63, doi: 10.1080/09084280701280502

92. Dubois B, Slachevsky A, Litvan I, Pillon B. The FAB: a frontal assessment battery at bedside. Neurology. (2000) 55:16216. doi: 10.1212/WNL.55.11.1621

93. Llinàs-Reglà J, Vilalta-Franch J, López-Pousa S, Calvó-Perxas L, Torrents Rodas D, Garre-Olmo J. The trail making test. Assessment. (2017) 24:18396. doi: $10.1177 / 1073191115602552$

94. Arbuthnott K, Frank J. Trail making test, part B as a measure of executive control: validation using a set-switching paradigm. J Clin Exp Neuropsychol. (2000) 22:518-28. doi: 10.1076/1380-3395(200008)22:4;1-0;FT518

95. Smith A. Symbol Digits Modalities Test: Manual. Los Angeles, CA: Los Angeles Western Psychological Services (1982).

96. Kaufman AS. Test review: Wechsler, D. Manual for the wechsler adult intelligence scale, revised. New York: Psychological Corporation, 1981. J Psychoeduc Assess. (1983) 1:309-13. doi: 10.1177/073428298300100310
97. Zarino B, Crespi M, Launi M, Casarotti A. A new standardization of semantic verbal fluency test. Neurol Sci. (2014) 35:140511. doi: 10.1007/s10072-014-1729-1

98. VolBrain. Automated MRI Brain Volumetry System. Available online at: https://www.volbrain.upv.es/ (accessed July 22, 2021)

99. Fazekas F, Chawluk J, Alavi A, Hurtig H, Zimmerman R. MR signal abnormalities at $1.5 \mathrm{~T}$ in Alzheimer's dementia and normal aging. Am J Roentgenol. (1987) 149:351-6. doi: 10.2214/ajr.149.2.351

100. Scheltens P, Barkhof F, Leys D, Pruvo JP, Nauta JJP, Vermersch P, et al. A semiquantative rating scale for the assessment of signal hyperintensities on magnetic resonance imaging. J Neurol Sci. (1993) 114:712. doi: 10.1016/0022-510X(93)90041-V

101. Staals J, Makin SDJ, Doubal FN, Dennis MS, Wardlaw JM. Stroke subtype, vascular risk factors, and total MRI brain small-vessel disease burden. Neurology. (2014) 83:1228-34. doi: 10.1212/WNL.00000000000 00837

102. Prins ND, van Straaten ECW, van Dijk EJ, Simoni M, van Schijndel RA, Vrooman HA, et al. Measuring progression of cerebral white matter lesions on MRI: Visual rating and volumetrics. Neurology. (2004) 62:15339. doi: 10.1212/01.WNL.0000123264.40498.B6

103. Lemieux J, Beaton DE, Hogg-Johnson S, Bordeleau LJ, Goodwin PJ. Three methods for minimally important difference: no relationship was found with the net proportion of patients improving. J Clin Epidemiol. (2007) 60:448-55. doi: 10.1016/j.jclinepi.2006.08.006

104. Maxwell SE. Sample size and multiple regression analysis. Psychol Methods. (2000) 5:434-58. doi: 10.1037/1082-989X.5.4.434

105. Del Brutto OH, Mera RM, Del Brutto VJ, Castillo PR. Lack of association between periodic limb movements during sleep and neuroimaging signatures of cerebral small vessel disease in strokefree community-dwelling older adults. The Atahualpa Project. J Stroke Cerebrovasc Dis. (2020) 29:104497. doi: 10.1016/j.jstrokecerebrovasdis.2019. 104497

106. Kim H, Thomas RJ, Yun C-H, Au R, Lee SK, Lee S, et al. Association of mild obstructive sleep apnea with cognitive performance, excessive daytime sleepiness, and quality of life in the general population: The Korean Genome and Epidemiology Study (KoGES). Sleep. (2017) 40(5). doi: 10.1093/sleep/zsx012

107. Salorio CF, White DA, Piccirillo J, Duntley SP, Uhles ML. Learning, memory, and executive control in individuals with obstructive sleep apnea syndrome. J Clin Exp Neuropsychol. (2002) 24:93-100. doi: 10.1076/jcen.24.1. 93.973

108. Redline S, Strauss ME, Adams N, Winters M, Roebuck T, Spry K, et al. Neuropsychological function in mild sleep-disordered breathing. Sleep. (1997) 20:160-7. doi: 10.1093/sleep/20.2.160

Conflict of Interest: The authors declare that the research to be conducted has no commercial or financial relationships that could be construed as a potential conflict of interest.

Publisher's Note: All claims expressed in this article are solely those of the authors and do not necessarily represent those of their affiliated organizations, or those of the publisher, the editors and the reviewers. Any product that may be evaluated in this article, or claim that may be made by its manufacturer, is not guaranteed or endorsed by the publisher.

Copyright (C) 2021 Spektor, Fietze and Poluektov. This is an open-access article distributed under the terms of the Creative Commons Attribution License (CC BY). The use, distribution or reproduction in other forums is permitted, provided the original author(s) and the copyright owner(s) are credited and that the original publication in this journal is cited, in accordance with accepted academic practice. No use, distribution or reproduction is permitted which does not comply with these terms. 